\title{
Alternation of the flickering morphology between the high and low state in MV Lyrae
}

\author{
A. Dobrotka ${ }^{1}, \mathrm{H} . \mathrm{Negoro}^{2}$, and P. Konopka ${ }^{3}$ \\ 1 Advanced Technologies Research Institute, Faculty of Materials Science and Technology in Trnava, \\ Slovak University of Technology in Bratislava, Bottova 25, 91724 Trnava, Slovakia \\ e-mail: andrej.dobrotka@stuba.sk \\ 2 Department of Physics, Nihon University, 1-8 Kanda-Surugadai, Chiyoda-ku, Tokyo 101-8308, Japan \\ 3 Komenského, 4530/6, 92101 Piešt' any, Slovakia
}

Received 28 March 2019 / Accepted 25 June 2020

\begin{abstract}
Aims. We studied unique data of the nova-like system MV Lyr taken by the Kepler space telescope during its transition from the high to low state and vice versa. We were interested in the evolution of frequency components found previously in different data also obtained by Kepler.

Methods. We divided the light curve into ten-day segments and investigated the corresponding power density spectra. We searched for individual frequency components by fitting with Lorentzian functions. Additionally, we investigated the variability using averaged shot profiles calculated from the light curve divided into ten equally spaced subsamples.

Results. We found very complex changes in the power density spectra. We focused our study onto three frequency components. A significant increase in activity is seen at low frequencies. Contrariwise, the high frequency part of the spectrum strongly decreases in power with a specific rise in characteristic frequencies of the individual components. We discuss various scenarios of this phenomenology such as the reprocessing of X-rays in a receding accretion disk or radiation from a more active region at the outer disk. Finally, we show that various cataclysmic variables show similar characteristic frequencies in their power density spectra. These are dependent on activity stage, making the situation similar to X-ray binaries.
\end{abstract}

Key words. accretion, accretion disks - stars: dwarf novae - stars: individual: MV Lyr - novae, cataclysmic variables $\mathrm{X}$-rays: binaries

\section{Introduction}

A great variety of objects, such as cataclysmic variables (CVs), are powered by the accretion process. This process is based on mass loss from a companion star. The transported gas falls toward the central compact object - a white dwarf - and in the absence of a strong magnetic field, an accretion disk forms. The same model is also valid for related objects like X-ray binaries (see, e.g., Warner 1995 or Frank et al. 1992 for a review). The main difference is that the latter has a neutron star or a black hole instead of a white dwarf.

The family of CVs is divided into several subclasses based on characteristic variability patterns. Dwarf novae show quasiregular outbursts with durations of several days and they appear on a timescale of 10-100 days (see Warner 1995 for review). Novalike or VY Scl systems spend most of their lifetime in a high state while they sporadically exhibit transitions into a low brightness state. This behavior can be connected to changes in accretion rate. In this way, the duration of the high states in VY Scl systems and dwarf novae is significantly different. It is relatively stable and long lasting for the former, while it is only temporary for the latter (outbursts). The shorter durations of the dwarf novae high state can be explained by the limited amount of gas in the depletive disk driving the outburst. The latter is a consequence of the mass accretion rate being unstable because of hydrogen ionization. This phenomenon is well explained by the so-called disk instability model (see Lasota 2001 for review). Meanwhile, in VY Scl systems, the mass accretion rate remains above the critical limit required for stability, explaining the longer duration of high states. The low state is generated by a sudden drop or stop of mass transfer from the secondary (King \& Cannizzo 1998; Hessman 2000).

The existence of the accretion process is usually seen as fast stochastic variability (also known as flickering). Flickering has three very basic observational characteristics; (1) linear correlation between variability amplitude and log-normally distributed flux (so-called rms-flux relation) observed in all varieties of accreting systems (see, e.g., Scaringi et al. 2012b; Van de Sande et al. 2015 for CVs case), (2) character of red noise or band limited noise with characteristic frequencies in power density spectra (PDS, see, e.g., Scaringi et al. 2012a; Dobrotka et al. 2014, 2016 for CVs case), and (3) time lags where flares reach their maxima slightly earlier in the blue than in the red (Scaringi et al. 2013; Bruch 2015)

An alternative method to "see" the PDS components was proposed by Negoro et al. (1994). The authors superposed many flares (shots) from the Ginga light curve of the X-ray binary Cyg X-1 in order to get a mean profile showing all typical stable features, like the central spike and the two sidelobes on both sides of the spike. A very similar profile was found by Sasada et al. (2017) using Kepler data of the blazar W2R $1926+42$, but on a much longer timescale. The third object showing such a multicomponent flare shape with a central spike and side-lobes is MV Lyr (Dobrotka et al. 2019) with a timescale 
between the X-ray binary and the blazar. All three findings make the situation very interesting because of the very different nature of all three objects. The radiation origin in the blazar in particular is very different from the other two objects.

MV Lyr is a well-studied, bright nova-like system ideal for a flickering study consisting of two phases, namely before and after the Kepler space telescope. During the former, several studies suggested various variability components. Besides coherent frequencies close to $\log (f / \mathrm{Hz})=-4.08$, Borisov (1992) and Skillman et al. (1995) reported a possible quasi-periodic oscillation (QPO) at approximately $\log (f / \mathrm{Hz})=-3.45$. Kraicheva et al. (1999) also found a QPO at a similar frequency. The first detection of the linear rms-flux relation was presented by Boeva et al. (2011). The latter attained an unambiguous shape using extensive Kepler data in the study of Scaringi et al. (2012b). Scaringi et al. (2012a) used the same Kepler data to study the PDS morphology. The authors reported a multicomponent nature with four different characteristic frequencies. Some of these components were close to the previously detected signals. A study of the time lags in different bands was performed by Scaringi et al. (2013) using ULTRACAM on the William Herschel Telescope. For MV Lyr $3 \mathrm{~s}$ lags were observed at the lowest frequencies with redder bands lagging behind the bluer ones. The authors suggest reprocessing of the X-ray photons on to the accretion disk or inside-out shocks traveling within the disk as explanations for the observed lags. Scaringi (2014) presented a sandwich model as responsible for the highest PDS component in which the central geometrically thin disk is surrounded by a geometrically thick disk (hot corona). This model was confirmed by direct X-ray observations by Dobrotka et al. (2017).

The localization of the individual PDS components is not trivial and various attempts have been made. Besides the modeling by Scaringi (2014), a shot noise model by Dobrotka et al. (2015) yields very similar results to the former: a radius of the highest frequency component emission region of approximately $10^{10} \mathrm{~cm}$, and a high $\alpha$ parameter (Shakura \& Sunyaev 1973) value (close to 1 ). Moreover, supposing $\alpha$ values from 0.1 to 0.4 , the two lower PDS components were localized into the geometrically thin disk and a more active outer disk rim.

The shot noise model is too strong a simplification of the real physics, and the localization of the PDS components, except the highest one based on Scaringi (2014) and Dobrotka et al. (2017), is still not certain. Therefore, observational studies are very useful, mainly during the dynamic stages of the accretion disk. Kepler data of MV Lyr comprise such a transition to the low state (and back) typical for nova-like systems (probably) caused by disk reformation. Therefore, we can follow the disk regression and subsequent reconstruction together with PDS evolution during such a state transitions. This can yield an identification of different flickering sources and a better understanding of the accretion flow geometry or structure of the accretion disk. In this paper we perform a detailed analysis of the mentioned activity stage transition of MV Lyr observed by the Kepler satellite.

\section{Data}

The data analyzed in this work represent the evolution of MV Lyr from the most common high state to a minimum and back. The data are taken by the Kepler satellite (Borucki 2010) with a cadence of approximately $60 \mathrm{~s}$. The studied light curve lasts approximately 585 days. Kepler data comprise a relatively large number of rare null point, which we removed. No other correction was needed. The bottom panel of Fig. 1 depicts this light

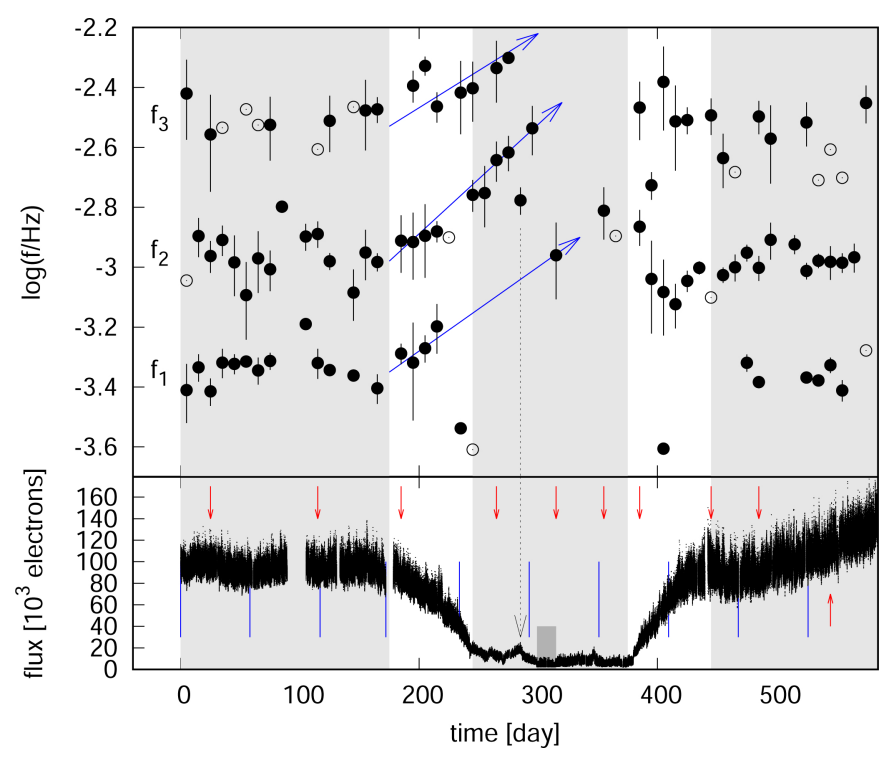

Fig. 1. Upper panel: time evolution of PDS components derived using Lorentzians in Eq. (1). The characteristic frequencies $f_{\mathrm{i}}$ (marked as labels on the left side) are shown by the black circles with error bars $\left(\delta \log \left(f_{\mathrm{i}}\right)\right)$ for significantly detected components with $\delta \log \left(f_{\mathrm{i}}\right)<0.2$, and by black circles without error bars for less significantly detected ones with $0.2<\delta \log \left(f_{\mathrm{i}}\right)<0.5$. The light shaded areas show time intervals selected as before, during and after the low state. Blue arrows outline possible frequency evolution, and the dashed vertical black arrow shows the connection of one deviated point with local re-brightening (see text for details). Lower panel: analyzed light curve for comparison. The red arrows show the (middle) time location of the PDS examples in Fig. 4. The dark shaded area is the deep low state identified by Scaringi et al. (2017) (see Sect. 4 for details). The vertical blue lines divide the light curve into ten equally spaced subsamples for the shot profile calculation in Sect. 4.

curve divided into three intervals marked as shaded areas showing before, during, and after the low state.

\section{PDS analysis}

Based on our motivation to study the PDS evolution from the high to the low state (and vice versa), we divided the light curve from Fig. 1 into equally spaced segments each with a duration of ten days. This allowed us to study any PDS changes with a time resolution of ten days.

\subsection{Method}

Every ten day segment is divided into ten subsamples, and a periodogram is calculated from each subsample using the Lomb-Scargle algorithm $^{1}$ (Scargle 1982). All periodograms (power ${ }^{2} p$ as function of frequency $f$ ) were subsequently transformed into $\log (f)-\log (p)$ space, for several reasons. Firstly, Papadakis \& Lawrence (1993) concluded that for an examination of variations of the PDS with time (to detect QPOs), periodogram averaging in logarithm is better than averaging the

\footnotetext{
1 Kepler data are full of gaps, rare null points, and the intervals of these null points. Therefore, the Lomb-Scargle method is suitable because it is designed for such unevenly spaced data. Otherwise a discrete Fourier transform would be ideal.

2 We use power normalized by the total variance according to Horne \& Baliunas (1986). Since the normalization does not affect the shape of the PDS, this has no importance in our case.
} 
periodograms themselves. Secondly, such averaging of $\log (p)$ yields symmetric errors (see, e.g., van der Klis et al. 1989; Aranzana et al. 2018). Thirdly, the whole $\log (f)$ interval of the PDS is re-binned with a constant frequency step of 0.05 , and such frequency bins comprise more and more periodogram points toward higher frequencies. The PDS scatter rises toward higher frequencies when binned with a constant frequency step in a linear scale (Fig. 4 of Scaringi et al. 2012a), and any potential frequency component above $\log (f / \mathrm{Hz})=-3$ is totally buried and non-detectable. Finally, all $\log (p)$ points within each frequency bin were averaged ${ }^{3}$, with the standard error of the mean as an uncertainty estimate. Such a PDS estimate has equal resolution in the whole $\log (f)$ interval (see, e.g., Shahbaz et al. 2005; Aranzana et al. 2018).

The periodogram averaging and binning lower the PDS scatter, while the light curve division determines the frequency resolution since it is equal to the lowest PDS frequency (before re-binning). The latter is proportional to the shortest light curve subsample duration. Therefore, an empirical compromise between noise and resolution must be found. The high frequency PDS end is set up empirically. Usually it is equal to the frequency where Poisson noise becomes dominant so that the PDS becomes flat (white noise) with no additional information. We concentrated our study on the high frequency part of the PDS, meaning we excluded the two lowest (from a total of four) PDS components detected by Scaringi et al. (2012a) because of the low frequency resolution of the PDS. The two remaining high frequency components were also detected in X-rays by Dobrotka et al. (2017), facilitating any discussion and interpretation. We also searched for an additional high frequency component that is also of X-ray origin (Dobrotka et al. 2019).

\subsection{Fitted model}

Every PDS is fitted with a multicomponent model using GNUPLOT $^{4}$. We used the same model as Scaringi et al. (2012a), but applied it in a slightly different way. The model consists of $n$ Lorentzian functions taken from Belloni et al. (2002),

$p=\sum_{i=1}^{n}\left[\frac{c_{\mathrm{i}} \Delta_{\mathrm{i}}}{\pi} \frac{1}{\Delta_{\mathrm{i}}^{2}+\left(f-f_{\mathrm{i}}\right)^{2}}\right]$,

where $c_{\mathrm{i}}$ is the constants, $f_{\mathrm{i}}$ is the searched characteristic frequency of the corresponding PDS components, and $\Delta_{\mathrm{i}}$ is its half width at half maximum. All PDSs were fitted with $n=4$ if possible. If any component was not identified visually, or the fitting process did not yield a satisfying result, we reduced the number of components up to $n=2$. Usually the fourth (lowest) Lorentzian describing the lowest frequency part converged to a very low value of $f_{\mathrm{i}}$ and depicted the rising or constant power toward the lowest frequencies. We are not interested in this component and it is simply used to describe continuously the whole PDS (Fig. 2).

Finally, when fitting the PDSs we used the averaged $\log (p)$ instead of $p$ (described above). All subsequent PDSs are better visualized in $\log (f \times p)$ as $y$-axis units. Such a visualization is suitable in the case of steep red noise, where individual components are better seen, which simplifies the identification of char-

\footnotetext{
If the number of averaged $\log -\log$ periodogram points per bin is lower than the selected value of 30 (three points from each of the ten periodograms), the bin is made larger until the condition is fulfilled. This happens for the lowest frequencies. The motivation is to get enough points for a mean value with error determination.

4 http://www.gnuplot.info/
}

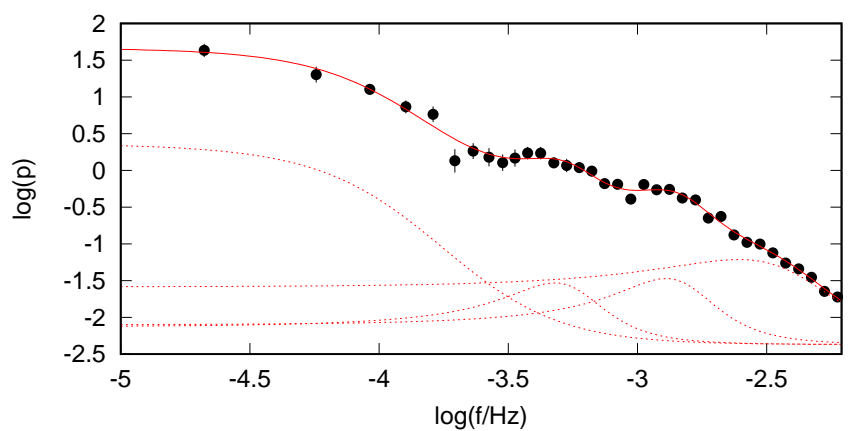

Fig. 2. Example of individual Lorentzian components (red dashed line) from Eq. (1) yielding a multicomponent shape of the fit (red solid line). While the fitting is performed in $\log (p)$ vs. $f$, the individual PDS "humps" are better seen in $\log (f \times p)$ vs. $\log (f)$ (see such an equivalent in Fig. 4, day 110-120).

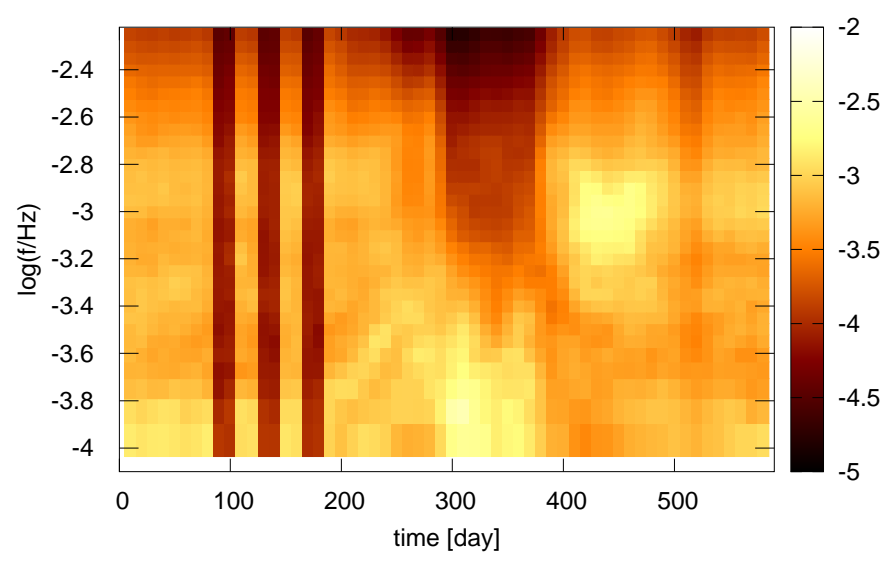

Fig. 3. Power density spectra evolution as a function of time with $y$-axis units of $\log (f \times p)$. The color and gray scale represents the normalized Lomb-Scargle power. The three vertical dark bands are gaps due to too large gaps in the data. The map is slightly smoothed.

acteristic frequencies needed for the number of components set up and the initial parameter estimate.

\subsection{Results}

The power of individual frequencies is depicted as an evolution map in Fig. 3, with some examples shown in Fig. 4. The decrease in the high frequency power during the low state is clear, while the power rises below approximately $\log (f / \mathrm{Hz})=-3.4$. Moreover, another strong power increase at approximately $\log (f / \mathrm{Hz})=-3$ after the minimum is noticeable too.

For further analysis and discussion of the PDS evolution we selected only the best and well-resolved fits of clear PDS components, meaning we selected PDS frequencies with errors $\delta \log \left(f_{\mathrm{i}}\right)<0.2$. All bad or poor fits yield large errors due to large PDS data scatter. Individual frequencies $f_{\mathrm{i}}$ are depicted in the upper panel of Fig. 1.

Three discrete frequencies before and after the minimum are noticeable. While these values seem to be stable during the high state, the frequencies are variable during the transition. During the latter the $f_{1}$ component is missing. As seen in Fig. 4, the PDS dramatically changes in shape in this dynamic stage, becoming steep with components that are difficult to resolve. Therefore, it is not sure whether the components have disappeared or their powers have just become too small to be resolved. Moreover, two 


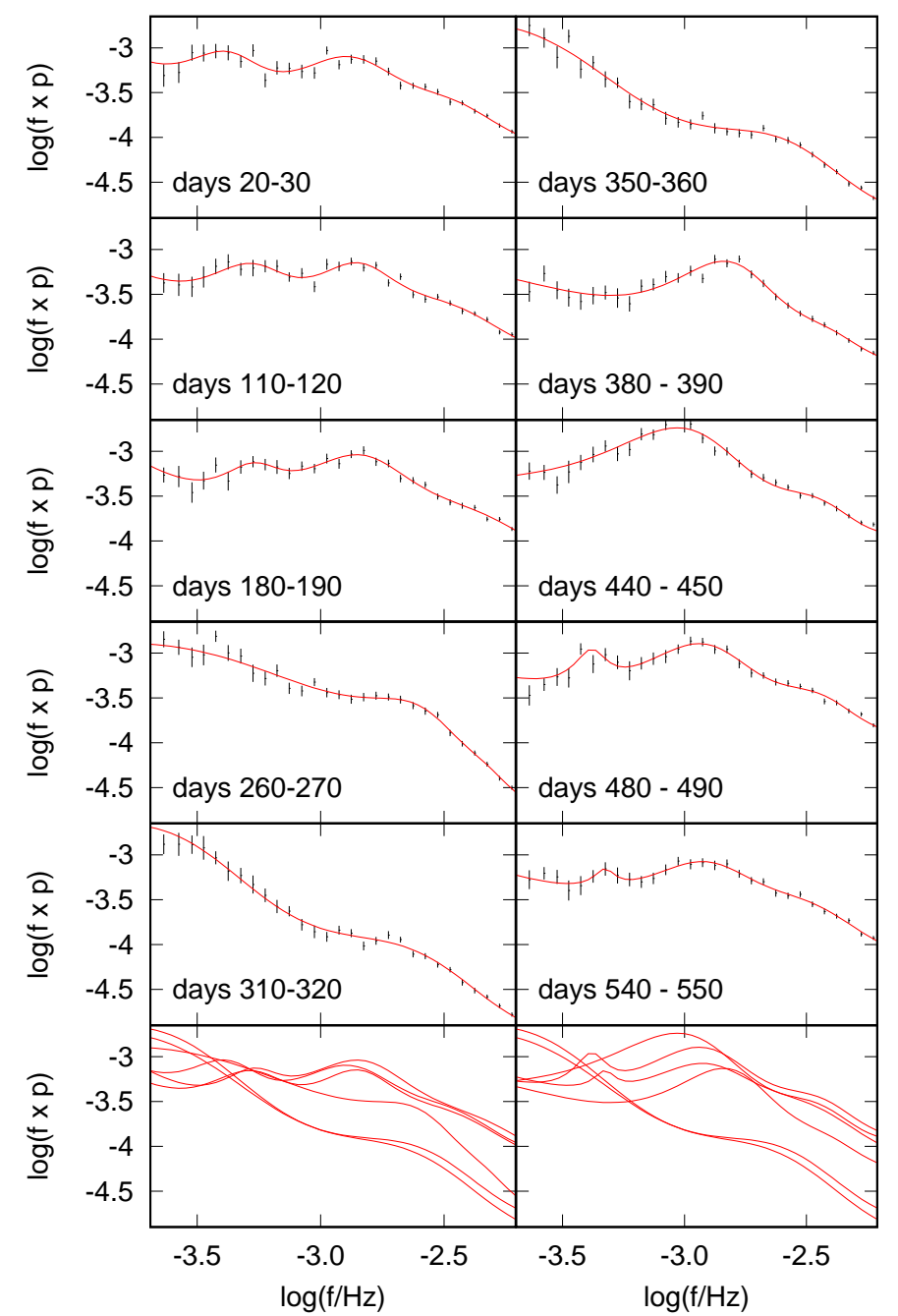

Fig. 4. Some representative examples of the calculated PDSs (small vertical lines as errors of the mean) with corresponding fits (red lines) in $f \times p$ as $y$-axis units. Two different cases are shown: transitions from the high to low state (left panels) and vice versa (right panels). The (middle) time locations of the PDSs are marked as red arrows in the lower panel of Fig. 1. All displayed fits are directly compared in the bottom panels (both panels comprise days 310-320 and 350-360 for comparison).

low frequency points at $\log (f / \mathrm{Hz})=-3.54$ and -3.61 (days 235 and 405 , respectively) appear during the transitions. Whether these belong to $f_{1}$ or are independent components is hard to conclude because of the small number of fitted frequencies. The evolution map in Fig. 3 is helpful, where a clear line is seen to rise from $\log (f / \mathrm{Hz})=-3.8$ to -3.6 between days 200 and 300. This suggests that the two deviated low frequency points represent an independent component with a frequency lower than $f_{1}$.

The distribution of frequencies before and after the transition is well described by histograms in Fig. 5. We fit individual cases with a multi-Gaussian model with three components. The fitted mean values with $1-\sigma$ as errors are summarized in Table 1 . Direct comparison of the fits (bottom panel of Fig. 5) suggests that after the minimum all frequencies gain their pre-minimum values.

We did not carry out the same histogram study for the low state because the points suggest variable behavior. Just at the beginning of the brightness fall, all three components start to deviate toward higher frequencies suggesting systematic variability (marked as blue arrows in Fig. 1). The trend continues

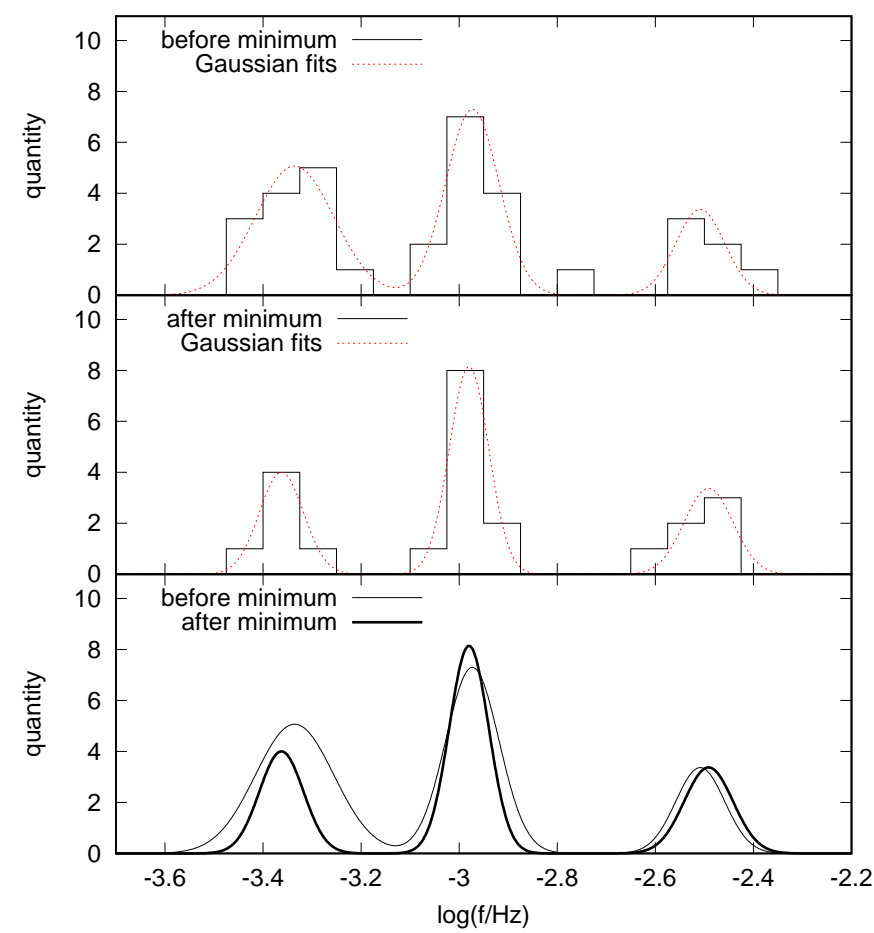

Fig. 5. Histograms of measured frequencies from the upper panel of Fig. 1 (points in the shaded areas are used). Multicomponent Gaussian fits are shown and compared in the bottom panel.

Table 1. Mean values with $1-\sigma$ parameters as error estimates of the frequencies from Fig. 1 before and after the low state (marked as gray areas in Fig. 1).

\begin{tabular}{lccc}
\hline \hline Position & $\log \left(f_{1} / \mathrm{Hz}\right)$ & $\log \left(f_{2} / \mathrm{Hz}\right)$ & $\log \left(f_{3} / \mathrm{Hz}\right)$ \\
\hline before & $-3.34 \pm 0.08$ & $-2.97 \pm 0.05$ & $-2.51 \pm 0.05$ \\
after & $-3.36 \pm 0.05$ & $-2.98 \pm 0.04$ & $-2.49 \pm 0.05$ \\
\hline
\end{tabular}

toward day 300 after which the detection of individual PDS components was very problematic or even impossible.

There are two interesting points during the low state in Fig. 1. One is at day 290. It does not follow the increasing trend of $f_{2}$, but jumps below other measurements. This point corresponds to a local re-brightening marked as a vertical dashed arrow. Probably this opposite brightness behavior corresponds to an opposite frequency evolution. The other deviated point is during day 320. It appears to be like a pre-minimum $f_{2}$ value, but it could also be a $f_{1}$ component that has evolved to such a high value (at the end of the corresponding blue arrow). After the low state, all frequencies return back to the pre-minimum level.

\section{Superposed shot profile analysis}

The use of PDS is a very generalized technique, and various structures with the same or a similar characteristic frequency can blend together and form a single PDS feature. This is the case of the dominant PDS feature at $\log (f / \mathrm{Hz})=-3$. Dobrotka et al. (2019) performed a superposed shot profile analysis (method based on Negoro et al. 1994) of the fast variability, and found that both the central spike and side lobes produce a PDS pattern very close to $\log (f / \mathrm{Hz})=-3$. If this PDS component is variable, 
the standard PDS study can not resolve substructures in which the shot profile is responsible for the variability.

\subsection{Method}

In light of this, as an additional study of the variability morphology we performed the same superposed shot profile analysis as Dobrotka et al. (2019). The goal was to "see directly" individual PDS components in the flare shape, like in the case of Cyg X-1 (Negoro et al. 2001). The method has three steps. First is the identification of shots and/or flares. A light curve point is identified as a peak if $N_{\text {pts }}$ points to the left and $N_{\text {pts }}$ points to the right have lower fluxes than the tested point.

In the second step, the flare extension must be defined, in this case as $N_{\text {ptsext }}$ points to the left and to the right from the peak. Like in Dobrotka et al. (2019), we used $N_{\text {ptsext }}=N_{\text {pts }} / 2$ in order not to superimpose a declining branch of one flare onto a rising branch of the adjacent flare, and vice versa.

In the last step after the flare selection, the flare points are averaged with maxima aligned, and the resulting averaged flux minimum is subtracted from all averaged points. All flares with rare individual null points or missing data (at the edge of the light curve or gaps) were excluded from the averaging process. As discussed in Dobrotka et al. (2019) the long-term trend and barycentric correction of Kepler data do not affect the result. As $N_{\text {pts }}$ we used a value of 50 . This results in 51 points (with a duration of approximately $58.8 \mathrm{~s}$ ) per flare yielding a flare duration of $3060 \mathrm{~s}$ with a corresponding frequency of $\log (f / \mathrm{Hz})=-3.49$. This is enough to visualize the studied frequency interval in the previous section.

For a superposed shot profile, we used ten equally spaced subsamples of the whole light curve as shown by the vertical blue lines in Fig. 1. This results in enough flares for averaging and we can visualize the rough time evolution. A larger number of light curve subsamples would yield a smaller number of flares per average resulting in higher noise and lower resolution.

Finally, Scaringi et al. (2017) investigated a part of the same data (during the low state) and found a quasi-periodic signal present only during the very faintest period of time when the light curve reaches a roughly constant minimum brightness level. The authors call this state a deep low state. They present a scenario in which this non-magnetic CV undergoes a short-lasting regime of magnetically driven accretion causing quasi-periodic bursts. In order not to contaminate the average profile with other burst-like patterns, we excluded the deep low state (between days 299 and 315) from the analysis. The QPOs have a shape of $30 \mathrm{~min}$ bursts repeating every two hours. The corresponding frequencies are $\log (f / \mathrm{Hz})=-3.86$ and -3.25 . The upper panel of Fig. 1 does not show any point at these frequencies during the problematic part, therefore the PDS is not affected.

\subsection{Results}

Resulting shot profiles with a central spike and side-lobes like in Dobrotka et al. (2019) are shown in Fig. 6. During the transition from the high to low state (upper right panel) all component amplitudes just decrease, with side-lobes being non-detectable at the end. During the transition from the low to high state (lower right panel) the behavior is reversed, and the central spike becomes higher in amplitude compared to the pre-minimum stage. While its amplitude increases and remains more or less stable after the transition, the amplitude of the side-lobes culminates toward the end of the transition phase (dotted line). The latter probably corresponds to the enhanced power of the $f_{2}$ com-
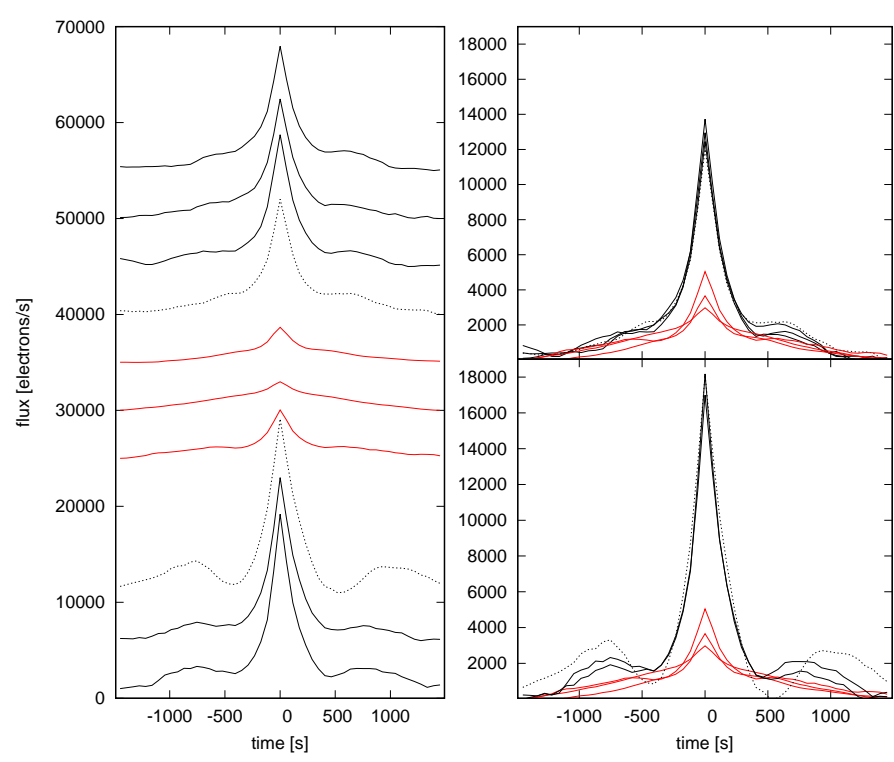

Fig. 6. Averaged shot profile time evolution. Black solid lines represent shot profiles during the high state, the black dotted lines are the profiles during both transitions, and the red solid lines represent the low state. Left panel: profiles vertically offset with time elapsing from up to down. Right panels: direct comparison and superposition of profiles showing accession to the low state (upper panel), and ascension from the low state (lower panel).

ponent at the end of the transition phase, seen in Figs. 3 or 4 as days 440-450. This is the information not provided by the standard PDS method: different shot profile substructures with the same or a similar characteristic frequency behaving differently blend into a single PDS component.

\section{Discussion}

We studied the PDS evolution of MV Lyr before, during, and after the low state captured by the Kepler satellite. We focused our study on frequencies detected in a previous PDS analysis by Scaringi et al. (2012a). We used a slightly different method to the authors in order to reduce the PDS scatter. We averaged and binned the periodograms in $\log -\log$ space with a constant logarithmic frequency step if a minimum number of averaged periodogram points per bin was satisfied. The latter reduces the resolution at low frequencies. Therefore, we were only interested in PDS components with the highest frequencies.

\subsection{Phenomenology}

All PDS components are probably present in both the high and low states, with variable values during the transition and low state. The two highest components show a significant increase in frequency. The lowest frequency component is difficult to detect in the low state, but if present it increases in frequency too. The power above $\log (f / \mathrm{Hz})=-3.4$ strongly decreases during the transition to the low state, while it significantly increases for lower frequencies. This different behavior suggests a different physical origin of the variability below and above $\log (f / \mathrm{Hz})=-3.4$.

The characteristic frequency variability during the transition suggests a correlation of the frequencies with the flux. However, after the system returns to the high state, the flux rises continuously while the frequency values are stable. Therefore, the 
frequencies depend on the activity stage and not the flux. This may be expected, because if the disk is fully reconstructed after the low state, no additional structural changes are generated by the increasing mass accretion rate. The idea is that the characteristic frequencies depend on the accretion flow structures (reconstructed and stable after the minimum), while the flux is proportional to the mass accretion rate (still rising after the minimum).

\subsection{Varying inner disk radius}

The presence of PDS components in various accreting systems motivates many authors to connect the frequency values with the physical parameters of that system. For example, the connection of black hole mass with a PDS break frequency in active galactic nuclei is such a case (see, e.g., González-Martín \& Vaughan 2012; Mohan \& Mangalam 2014). This should be the result of different inner disk radii being a function of the black hole mass. In CVs the PDS components can be connected to the inner disk radius too (Balman \& Revnivtsev 2012). The luminosity of these binaries is dominated by the release of the gravitational potential energy of the gas in the disk, and therefore the brightness is directly connected to the mass accretion rate. The sudden drop in brightness in MV Lyr or VY Scl binaries (see Warner 1995 for a review) means that the mass accretion rate decreases considerably. The mass transfer can fall from more than $10^{-8} M_{\odot} \mathrm{y}^{-1}$ to less than $10^{-11} M_{\odot} \mathrm{y}^{-1}$ (Scaringi et al. 2017). The latter is typical for a dwarf novae in quiescence. Following the disk instability model (see Lasota 2001 for a review), the disk is truncated during such a low state, while it is fully reformed down to the white dwarf surface in the high state. Therefore, during the transition from the high to low state studied in this paper, the inner disk must recede to larger radii. This is the basic idea of Balman \& Revnivtsev (2012) who suggest that the characteristic frequency of a PDS component generated at the inner disk radius must decrease simultaneously. However, the variability of all components in this paper shows the opposite behavior. This suggests that the mentioned correlation of the characteristic frequencies with the inner disk radius is not applicable.

\subsection{Characteristic frequencies $f_{2}, f_{3}$ and reprocessing model}

Another explanation of the observed behavior is based on the model proposed by Scaringi (2014). Following the author, the $f_{2}$ component is generated by an inner geometrically thick disk, the so-called X-ray corona. This disk radiates X-rays, which are subsequently reprocessed into optical radiation by the inner geometrically thin disk. Dobrotka et al. (2017) confirmed the $\mathrm{X}$-ray origin of this PDS component, which supports a corona origin. Moreover, the $f_{3}$ component is of X-ray origin too (Dobrotka et al. 2019). If the thin disk is reprocessing the X-rays into optical, the recession of this disk during the low state means a lower reprocessing surface. This can result in an optical flux decrease while the frequencies are unchanged (Fig. 7). The variation of the latter can be generated by the transformation of the corona caused by a changing mass accretion rate.

Another aspect is the X-ray emissivity. During the high state only a small fraction of the flow evaporates into the corona. The mass accretion rate based on a cooling flow model yields only $7 \times 10^{-12} M_{\odot} \mathrm{y}^{-1}$ (Dobrotka et al. 2017). This is a typical value of the mass accretion rate in the low state of this class of objects (see, e.g., Gänsicke et al. 1999), when the central disk is truncated and the whole mass flow evaporates into the corona (Fig. 7). The X-rays are generated by free-free transitions and

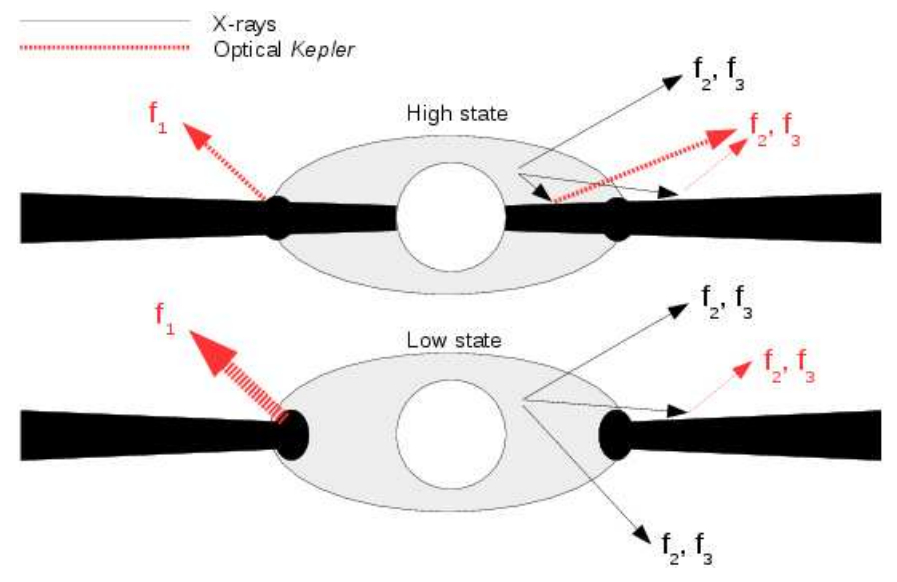

Fig. 7. Visualization of the proposed scenario, where $f_{2}$ and $f_{3}$ components are generated in the hot corona as X-rays that are reprocessed into optical radiation by the underlying thin disk. The thickness of the arrows represents the radiation intensity. The text gives details.

the emissivity is proportional to $n^{2}$, where $n$ is the plasma density. If the mass accretion rate (or $n$ ) through the corona is the same (unchanged) during both the high and low states, the gravitational energy liberation does not vary. This results in an unaltered amplitude of the variability, and no PDS power decrease due to emissivity is expected. Apparently, this is not observed as shown in this paper. Therefore, an additional physical process (such as reprocessing) affecting the variability is expected.

Finally, the temporal higher power of the $f_{2}$ component after the minimum is interesting. During the disk redevelopment the propagating increase of the mass accretion from outer disk regions pushes the inner disk edge toward the white dwarf. Such a mass wave can enhance specific variability in the corona, which returns to the initial pre-minimum stage after relaxation and stabilization of the flow.

\subsection{Energetic aspect of the reprocessed $X$-rays}

The reprocessing scenario is attractive, but an energy-based test is worth doing. For this purpose we used the XMM-Newton OM data presented in Dobrotka et al. (2017) because these data are well calibrated, while the Kepler case is more problematic. After transforming the OM light curve taken with the UVW1 filter into fluxes, the amplitude of the variability represented by the root square of the variance is of $2.3 \times$ $10^{-14} \mathrm{ergs} \mathrm{s}^{-1} \mathrm{~cm}^{-1} \AA^{-1}$. Assuming the same flux in the whole optical band we get a rough estimate of the integrated optical flux. However, this is merely an upper limit because it is well known that the flickering has a higher amplitude toward higher energies. So in reality, the amplitude should decrease toward lower energies. Let us suppose that the wavelength interval of our assumed "optical" band is between 2500 and $7000 \AA$ (limits of $\mathrm{UVW}^{5}$ and $\mathrm{V}$ filter). Multiplying the UVW1 flux by the length of this band $(4500 \AA)$ we get the integrated flux of $1.4 \times 10^{-10} \mathrm{ergs} \mathrm{s}^{-1} \mathrm{~cm}^{-1}$, that is, due to the roughness of the estimate we take $\sim 10^{-10} \operatorname{ergs~s}{ }^{-1} \mathrm{~cm}^{-1}$. Moreover, Dobrotka et al. (2017) mention that the Swift flux in the interval of $0.2-10 \mathrm{keV}$ is approximately $5.4 \times 10^{-12} \mathrm{ergs} \mathrm{s}^{-1} \mathrm{~cm}^{-1}$, which is approximately 20 times less than the estimated optical flux upper limit. This makes the reprocessing scenario energetically insufficient.

\footnotetext{
Using lower energy filters like U or B starting at 3000 or $3500 \AA$ instead of UVW1 does not change the following energetic discussion.
} 


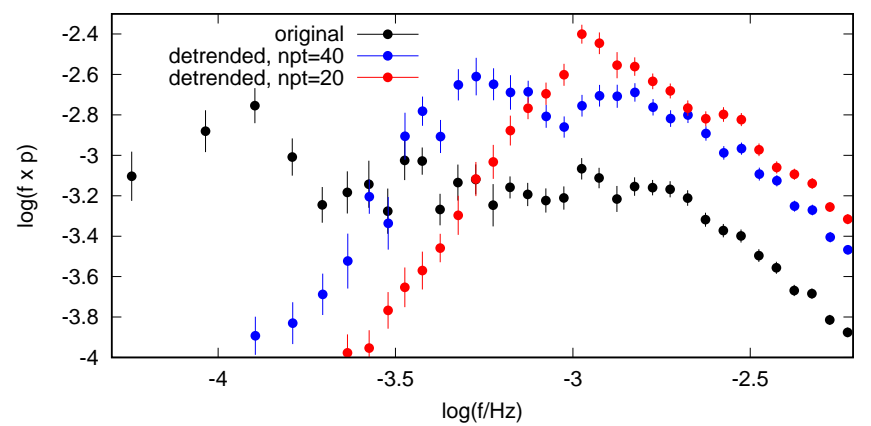

Fig. 8. Power density spectra from the first ten days of Kepler data. We compare the original case with two PDSs calculated from data after detrending using the running median. The number of median window points is represented by $n p t$.

We do not expect that the radiation outside the $0.2-10 \mathrm{keV}$ range contributes significantly to the overall X-ray flux. Therefore, the estimated X-ray flux is at its maximum. In order to get both energy bands closer, a more realistic estimate of the optical flux is the only possibility. Some reduction of the latter can be achieved when accounting for decreasing flux toward lower energies. If we assume that the flickering amplitude decreases linearly, and reaches a value of zero at the lower energy limit of the $\mathrm{V}$ filter ${ }^{6}$, the reduction factor is two. This does not resolve the energy insufficiency, it only decreases the band ratio from 20 to 10 .

Another aspect is the timescale of the flickering variability. Scaringi (2014) only modeled the $f_{2}$ frequency, and identified the corona as the variability source. Therefore, in such a case the reprocessing only generates the optical $f_{2}$ signal, and the lower frequency components can be generated by the accretion disk itself. Therefore, in order to get a correct energy estimate, the lower frequencies must be excluded from the flickering. For this purpose we detrended the OM light curve from Fig. 1 in Dobrotka et al. (2017) using a running median. Using 20 adjacent points ${ }^{7}$ for the running window from which the median is calculated, the $f_{2}$ feature is still dominant in the PDS. The corresponding amplitude of the variability is approximately 1.5 times lower. Such a reduction of the variability amplitude is again not a solution, and it only decreases the band ratio from 10 to approximately 7. A lower number of points per median window already affects the dominant $f_{2}$ PDS feature, and therefore any further reduction of the variability amplitude is not possible.

The Kepler case is negligibly better. In Fig. 8 we show the original and detrended PDSs of the first ten days. Detrending using 40 and 20 points per median window reduces the variability amplitude by a factor of approximately 1.8 and 2, respectively (Fig. 9). Apparently, all "attempts" to reduce the optical flux are insufficient, and all estimates are still one order of magnitude larger than the estimated X-ray flux.

Apparently, we have a serious inconsistency between interpretation and energies. The clue is in an exact understanding of the variability components. Relying only on PDS, we study one $f_{2}$ pattern. However, the shot profile studied in Sect. 4 and in Dobrotka et al. (2019) identify two $f_{2}$ components that blend and are indistinguishable in a standard PDS study. We propose a

\footnotetext{
6 This allows some amplitude to be observable also in the $R$ band.

7 Ten points to the left, ten to the right from the corrected point. This yields a timescale of $1050 \mathrm{~s}$ (including the corrected point) with the OM light curve cadence of $50 \mathrm{~s}$, which corresponds approximately to the frequency $f_{2}$.
}

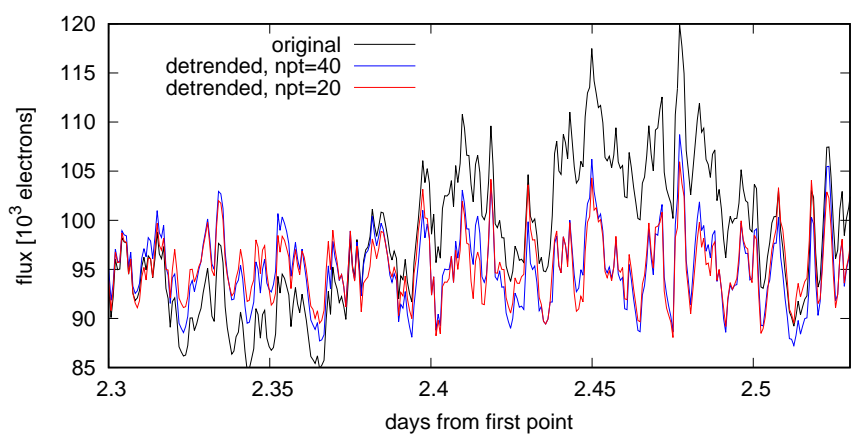

Fig. 9. Snapshot of the Kepler light curve compared with the detrended cases after removing the running median. The low frequency variability not present in the red and blue curves is clear. The parameter $n p t$ has the same meaning as in Fig. 8.

model in which the inner disk mass accretion fluctuations are somehow correlated with the fluctuations in the corona. The inner disk generates its own high amplitude $f_{2}$ pattern, while the corona generates a low amplitude $f_{2}$ pattern by reprocessing. Such a high amplitude pattern could be the central spike seen in Fig. 6, while the low amplitude patterns could be the side lobes. Inspecting the amplitudes from Fig. 5 in Dobrotka et al. (2019), we find that the side lobe amplitudes are lower than the peak one by factors of 8 and 9.2 for the left and right side lobe, respectively. Taking the profiles from Fig. 6, the factors are comparable except the one with clearly enhanced side lobes. This reduces the corresponding optical flux by one order of magnitude, making the energetic ratio satisfied and suitable for the reprocessing scenario.

Finally, the corona is an evaporation of the underlying thin disk, and any mass accretion fluctuation of this thin disk must results in a modulation of the mass evaporation. Such fluctuations in evaporation can modulate and trigger mass accretion fluctuations in the corona. This is the above-assumed diskcorona correlation.

\subsection{Low frequency components}

The behavior of the $f_{1}$ component during the low state is enigmatic because of poor statistics. However, a strong power rise toward lower frequencies is noticeable. Whether it represents the shifting $f_{1}$ is not sure, but certainly it suggests increasing activity below $f_{1}$. Not reacting to inner disk recession like $f_{2}$ and $f_{3}$ suggests that the origin of this power rise must be looked for elsewhere. An outer disk region with possible enhanced activity during the low state must be a source. For example, the diskcorona interaction (Fig. 7) at the corona outer edge could be a solution.

Such a more active region could be a source of QPOs generated by rotational motion of accretion inhomogeneity. This rotation would generate variabilities on a dynamical timescale of

$t_{\text {dyn }} \sim\left(\frac{R^{3}}{G M_{1}}\right)^{1 / 2}$,

where $G$ is the gravitational constant, $M_{1}$ the primary mass, and $R$ the distance from the center. Using $M_{1}=0.73 M_{\odot}$ (Hoard et al. 2004) $t_{\mathrm{dyn}}=72$ or $580 \mathrm{~s}$ for $R=8 \times 10^{9}$ and $32 \times 10^{9} \mathrm{~cm}$, respectively. The former is the corona radius derived by Scaringi (2014), while the latter is a disk radius estimate from Dobrotka et al. (2015). Corresponding frequencies of $\log (f / \mathrm{Hz})$ 


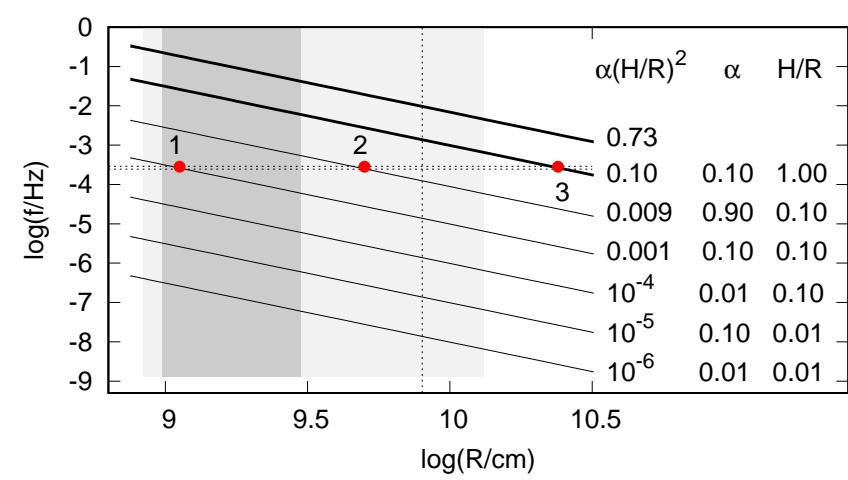

Fig. 10. Frequencies corresponding to viscous timescale compared to the two lowest measured frequencies from Fig. 1 (two horizontal dotted lines). Various models with different parameters listed as labels are shown. The vertical dotted line is the corona radius from Scaring (2014). The shaded areas are the inner disk radius intervals from Fig. 4 of Scaringi et al. (2017). Labeled points are solutions showing the measured frequencies.

$\sim-2.8$ and -1.9 are too high for $f_{1}$ or lower values. However, the value of -2.8 is close to $\log \left(f_{2} / \mathrm{Hz}\right) \sim-3.0$ component in the high state. However, from Scaringi (2014) and Dobrotka et al. (2017) we know that the viscous process in the geometrically thick X-ray corona is the probable source in this case.

Since we study the disk reconfiguration driven by viscous processes, a similar estimate can be made for the viscous timescale,

$t_{\mathrm{visc}} \sim \frac{t_{\mathrm{dyn}}}{\alpha(H / R)^{2}}$,

where $\alpha$ is the basic disk parameter by Shakura \& Sunyaev (1973) and $H$ is the height scale of the disk. Some examples of the corresponding frequencies as a function of $R$ are shown in Fig. 10. The values are calculated from the white dwarf surface estimated following Nauenberg (1972) up to the outer disk edge of $32 \times 10^{9} \mathrm{~cm}$. Various combinations of $\alpha$ and $H / R$ are shown. Apparently, compared to the horizontal dotted lines showing the frequencies of $\log \left(f_{1} / \mathrm{Hz}\right)=-3.54$ and -3.61 (lowest values from Fig. 1), a large range of $\alpha(H / R)^{2}$ is possible: from 0.001 to 0.1 shown as three solutions (points $1,2,3$ ) yielding $H / R \geq 0.1$. This suggests that the source is no longer a geometrically thin disk. The solutions range from (point 1) an inner radius disk derived by Scaringi et al. (2017) using the maximum possible constraints (dark shaded area in Fig. 10) up to (point 3) a radius larger than the coronal radius derived by Scaringi 2014. Therefore, we are not able to quantify the source characteristics, and the only conclusion is that the geometrically thick corona is a potential solution.

Other disk structures can still be candidates for the low frequency components. A hot spot or stream (from the secondary) overflow is also localized further away from the central disk. The superhump interpretation would be also possible, but the discussed frequencies of $\log (f / \mathrm{Hz})=-3.54$ and -3.61 are far from the superhump case of $\log (f / \mathrm{Hz})=-4.08$ derived by Borisov (1992) and Skillman et al. (1995). Only direct X-ray observation can provide a final answer. It is crucial to determine whether $f_{1}$ is of X-ray origin like $f_{2}$ and $f_{3}$, and what the PDS power is as compared to the high state. If detected in X-rays, this would exclude any stream overflow, hot spot, or superhump scenario, and would locate the radiation source in central regions like a corona or boundary layer.

\subsection{Analogy with $X$-ray binaries}

Knowledge of the presence of PDS components in Kepler data of $\mathrm{CVs}$ at frequencies close to the values determined in this paper is not new. Dobrotka \& Ness (2015) and Dobrotka et al. (2016) studied PDSs of two dwarf novae present in the Kepler field, V1504 Cyg and V344 Lyr. Both systems show the PDS behaving in a similar way depending on the activity stage. Surprisingly not only are the frequency values comparable, but their presence depending of the activity stage is also comparable. During the low state both systems show PDS components close to $f_{1}$, while during the high state other components close to $f_{2}$ are present.

Some other systems studied from the ground show similar low frequency components, such as KR Aur (Kato et al. 2002) and UU Aqr (Baptista \& Bortoletto 2008). While the former shows practically the same characteristic PDS frequency as derived in this paper, the latter is slightly lower ${ }^{8}$ reported a presence of spiral arm and this can significantly change the nature of the variability, i.e. it may differ from the "standard" case.. Balman \& Revnivtsev (2012) reported several high frequency components close to $f_{3}$ in X-ray data of SS Cyg, RU Peg, VW Hyi, WW Cet, and T Leo. Dobrotka et al. (2014) reanalyzed the observation of the dwarf nova RU Peg during quiescence and found the multicomponent nature of the PDS with similar characteristic frequencies in UV and X-rays. Contrary to V1504 Cyg and V344 Lyr, the quiescent $\mathrm{X}$-ray PDS also comprises a component between $f_{2}$ and $f_{3}$. Perhaps more or all components are present in both stages of $\mathrm{CVs}$ in general, but some remain very damped during the low state. After all, the $f_{2}$ and $f_{3}$ components in MV Lyr did not disappear during the low state, the power is simply very low. Because of the latter and the probable variability, the characteristic values are hard to determine.

All the mentioned values are summarized in Table 2 and Fig. 11. Three distinct groups are potentially present. However, confirmation of this statement requires a much larger statistical set of measured frequencies, because the histogram in Fig. 11 could be a result of a random process.

Such typical PDS components depending on brightness states are very typical for X-ray binaries (see, e.g., Miyamoto et al. 1992, 1993, 1994 as pioneering works, or Lewin \& van der Klis 2010 for a review), while they are new for CVs.

\section{Summary and conclusions}

The multicomponent PDS of MV Lyr shows a complex transformation during the transition from the high to low state and vice versa with three resolved PDS components. The high frequency part of the PDS $(\log (f / H z)>-3.4)$ considerably decreases in power during the minimum brightness compared to the high state, while the low frequency part $(\log (f / \mathrm{Hz})<-3.4)$ significantly increases. The characteristic frequencies of the two highest PDS components increase during the low state. The same is also possible for the lowest PDS component, but with a lower significance. Once the brightness starts to get back to the preminimum level, the frequencies return to their pre-minimum values. An additional transient power increase of the middle PDS component at $\log (f / H z) \simeq-3$ is clear after the minimum. The evolution of frequencies together with the overall flux suggests that the frequency values are correlated with the brightness state, and not with the flux.

\footnotetext{
8 Baptista \& Bortoletto (2008)
} 
Table 2. Approximate characteristic PDS frequencies in different CVs systems used for Fig. 11.

\begin{tabular}{llcc}
\hline \hline System & State & Band & $\log (f / \mathrm{Hz})$ \\
\hline MV Lyr $^{(a)}$ & Low & Optical & $-3.5,(-2.5)$ \\
& Low & Optical & $-3.6,(-2.3)$ \\
V1504 Cyg $^{(b)}$ & Low & Optical & $-3.4,-2.3$ \\
V344 Lyr $^{(c)}$ & Low & Optical & -3.4 \\
RU Peg $^{(d)}$ & Low & X-rays & $-3.5,-2.7$ \\
& Low & UV & -3.3 \\
SS Cyg $^{(e)}$ & Low & X-rays & -2.3 \\
& Low & X-rays & -2.4 \\
VW Hyi $^{(e)}$ & Low & X-rays & -2.7 \\
WW Cet $^{(e)}$ & Low & X-rays & -2.5 \\
T Leo $^{(e)}$ & Low & X-rays & -2.4 \\
\hline MV Lyr $^{(a)}$ & High & Optical & $-3.3,-3.0,-2.5$ \\
& & & -3.4 \\
V1504 Cyg $^{(b)}$ & High & Optical & $-3.3,-3.0,-2.9,-2.4$ \\
V344 Lyr $^{(c)}$ & High & Optical & $-3.5,-3.0,-2.8$ \\
KR Aur $^{(f)}$ & High & Optical & -3.4 \\
UU Aqr $^{(g)}$ & High & Optical & -3.8 \\
\hline
\end{tabular}

Notes. The values in parenthesis are maximal (not necessary final) values of $f_{2}$ and $f_{3}$ from Fig. 1 . We did not add the hypothetical highest value of $f_{1}$ (in the low state) because it is too speculative. ${ }^{(a)}$ This paper. ${ }^{(b)}$ From Table 2 of Dobrotka \& Ness (2015). ${ }^{(c)}$ From Table 2 of Dobrotka et al. (2016). ${ }^{(d)}$ From Dobrotka et al. (2014) ${ }^{(e)}$ From Balman \& Revnivtsev (2012). ${ }^{(f)}$ From Kato et al. (2002). ${ }^{(g)}$ From Baptista \& Bortoletto (2008).

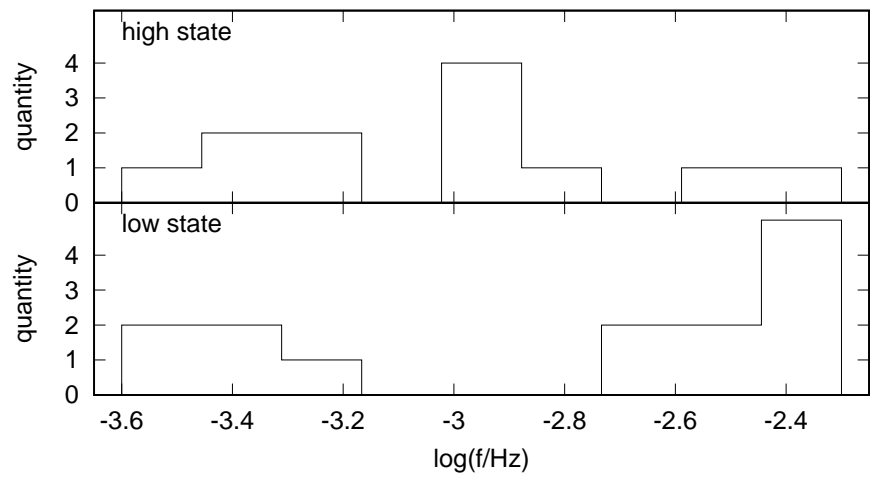

Fig. 11. Histograms of the values from Table 2. The value of -3.8 was excluded because of spiral patterns in the disc of UU Aqr making the case "non standard".

The power decrease of the high frequency part of the PDS can be explained by the diminishing of the receding disk surface needed for X-ray reprocessing. This is based on the knowledge that the highest PDS components are generated by the inner X-ray corona or boundary layer. However, rough estimates of the integrated optical and X-ray fluxes imply that the reprocessing scenario can not work for the whole variability, but only for its low amplitude components. A rough timescale estimate implies that the source of the lowest frequencies could be a standard disk (geometrically thicker than the thin solution) embedded into the geometrically thick X-ray corona or the corona itself.
Finally, the comparison with other CV systems suggests that CVs may have common typical PDS components with similar values depending on the brightness state. This is similar to what is observed in X-ray binaries. However, a larger statistical set of measurements is needed to confirm or deny this hypothesis.

Acknowledgements. AD was supported by the Slovak grant VEGA 1/0408/20, and by the Operational Programme Research and Innovation for the project : Scientific and Research Centre of Excellence SlovakION for Material and Interdisciplinary Research, code of the project ITMS2014+ : 313011W085 co-financed by the European Regional Development Fund. HN was supported by Grants-inAid for Scientific Research 16K05301 from the Ministry of Education, Culture, Sports, Science and Technology (MEXT) of Japan. We acknowledge the anonymous referee for helpful comments, particularly concerning the energetic study of the optical and X-ray flickerings.

\section{References}

Aranzana, E., Körding, E., Uttley, P., Scaringi, S., \& Bloemen, S. 2018, MNRAS, 476, 2501

Balman, Ş., \& Revnivtsev, M. 2012, A\&A, 546, A112

Baptista, R., \& Bortoletto, A. 2008, ApJ, 676, 1240

Belloni, T., Psaltis, D., \& van der Klis, M. 2002, ApJ, 572, 392

Boeva, S., Bachev, R., Tsvetkova, S., et al. 2011, BlgAJ, 16, 23

Borisov, G. V. 1992, A\&A, 261, 154

Borucki, W. J., Koch, D., Basri, G., et al. 2010, Science, 327, 977

Bruch, A. 2015, A\&A, 579, A50

Dobrotka, A., \& Ness, J.-U. 2015, MNRAS, 451, 2851

Dobrotka, A., Mineshige, S., \& Ness, J.-U. 2014, MNRAS, 438, 1714

Dobrotka, A., Mineshige, S., \& Ness, J.-U. 2015, MNRAS, 447, 3162

Dobrotka, A., Ness, J.-U., \& Bajčičáková, I. 2016, MNRAS, 460, 458

Dobrotka, A., Ness, J.-U., Mineshige, S., \& Nucita, A. A. 2017, MNRAS, 468, 1183

Dobrotka, A., Negoro, H., \& Mineshige, S. 2019, A\&A, 631, A134

Frank, J., King, A., \& Raine, D. 1992, Cambridge Astrophys. Ser., 21

Gänsicke, B. T., Sion, E. M., Beuermann, K., et al. 1999, A\&A, 347, 178

González-Martín, O., \& Vaughan, S. 2012, A\&A, 544, A80

Hessman, F. V. 2000, New Astron. Rev., 44, 155

Hoard, D. W., Linnell, A. P., Szkody, P., et al. 2004, ApJ, 604, 346

Horne, J. H., \& Baliunas, S. L. 1986, ApJ, 302, 757

Kato, T., Ishioka, R., \& Uemura, M. 2002, PASJ, 54, 1033

King, A. R., \& Cannizzo, J. K. 1998, ApJ, 499, 348

Kraicheva, Z., Stanishev, V., \& Genkov, V. 1999, A\&AS, 134, 263

Lasota, J. 2001, New Astron. Rev., 45, 449

Lewin, W., \& van der Klis, M. 2010, Compact Stellar X-ray Sources (Cambridge: Cambridge University Press)

Miyamoto, S., Kitamoto, S., Iga, S., Negoro, H., \& Terada, K. 1992, ApJ, 391, L21

Miyamoto, S., Iga, S., Kitamoto, S., \& Kamado, Y. 1993, ApJ, 403, L39

Miyamoto, S., Kitamoto, S., Iga, S., Hayashida, K., \& Terada, K. 1994, ApJ, 435, 398

Mohan, P., \& Mangalam, A. 2014, ApJ, 791, 74

Nauenberg, M. 1972, ApJ, 175, 417

Negoro, H., Miyamoto, S., \& Kitamoto, S. 1994, ApJ, 423, L127

Negoro, H., Kitamoto, S., \& Mineshige, S. 2001, ApJ, 554, 528

Papadakis, I. E., \& Lawrence, A. 1993, MNRAS, 261, 612

Sasada, M., Mineshige, S., Yamada, S., \& Negoro, H. 2017, PASJ, 69, 15

Scargle, J. D. 1982, ApJ, 263, 835

Scaringi, S. 2014, MNRAS, 438, 1233

Scaringi, S., Körding, E., Uttley, P., et al. 2012a, MNRAS, 421, 2854

Scaringi, S., Körding, E., Uttley, P., et al. 2012b, MNRAS, 427, 3396

Scaringi, S., Körding, E., Groot, P. J., et al. 2013, MNRAS, 431, 2535

Scaringi, S., Maccarone, T. J., D’Angelo, C., Knigge, C., \& Groot, P. J. 2017, Nature, 552, 210

Shahbaz, T., Dhillon, V. S., Marsh, T. R., et al. 2005, MNRAS, 362, 975

Shakura, N. I., \& Sunyaev, R. A. 1973, A\&A, 24, 337

Skillman, D. R., Patterson, J., \& Thorstensen, J. R. 1995, PASP, 107, 545

Van de Sande, M., Scaringi, S., \& Knigge, C. 2015, MNRAS, 448, 2430

van der Klis, M. 1989, in NATO Advanced Science Institutes (ASI) Series C,

eds. H. Ögelman, \& E. P. J. van den Heuvel, NATO Adv. Sci. Inst. (ASI) Ser.

C, 262, 27

Warner, B. 1995, Cambridge Astrophys Ser., 28 